\title{
A formation far, far away
}

Astrophys. J. (in the press); preprint available at https://arxiv.org/abs/1909.11246

We still have very limited knowledge of the elemental composition of giant planets. Our best example is currently Jupiter, thanks to Galileo's end-of-mission plunge into its atmosphere. Surprisingly, all elements (bar oxygen, whose depletion was probably due to especially dry local conditions) are enriched with respect to solar values by approximately the same amount - about a factor of three. This shouldn't happen if Jupiter were accreted around its current location ( $5 \mathrm{au})$ : only some elements (sulfur, phosphorus and possibly carbon) would have been in the solid phase in the solar nebula, whereas others (nitrogen and the noble gases) would have still been volatile and would have dispersed. Among the proposed solutions to this puzzle, the idea that Jupiter's core was formed further out in the Solar System, where nitrogen and various noble gases were frozen and could accrete, is gaining ground in theoretical and observational studies. Karin Öberg and Robin Wordsworth find that the enrichment pattern can indeed be explained if Jupiter's core formed beyond $\sim 30$ au.

Öberg and Wordsworth employ standard analytical power laws to determine the density and temperature profiles of the solar nebula, and actual data to set the location of snowlines of various molecules, allowing them to determine the abundance profile for both their gaseous and solid phases. They consider various contributions for accretion of materials onto Jupiter (core dissolution, pebbles and gas), exploring several combinations of these sources' accretion locations. Jupiter's composition from Galileo can be reproduced if its core formed beyond both the nitrogen and the argon snowlines. The authors also rule out a major role of entrapment of volatiles into ice pebbles (for example, in clathrate form), which has sometimes been evoked to reconcile Galileo's observations and an in situ formation of Jupiter's core.

Öberg and Wordsworth conclude with some predictions. If their model is correct, the bulk oxygen abundance in Jupiter should be enriched to almost the same level as the other elements. Saturn should also be enriched following a similar pattern, at least for most of the elements, even though the details could be connected to the specificities of Saturn's formation history. Cassini, which ended its mission with a similar plunge into Saturn as Galileo did for Jupiter, will provide some constraints.

\section{Luca Maltagliati}

Published online: 29 October 2019

https://doi.org/10.1038/s41550-019-0950-5 